\title{
Stress factors' effects on the induction of lipid synthesis of microalgae
}

\author{
Shakirov Zair Saatovich ${ }^{1 *}$, Khalilov Ilkhom Mamatkulovich ${ }^{1}$, Khujamshukurov Nortoji ${ }^{2}$ \\ ${ }^{1}$ Institute of Microbiology, Uzbekistan Academy of Sciences, Tashkent, Republic of Uzbekistan. \\ ${ }^{2}$ Tashkent Chemical-Technological Institute, Tashkent, Republic of Uzbekistan.
}

\section{ARTICLE INFO \\ Article history: \\ Received on: May 14, 2021 \\ Accepted on: July 18, 2021 \\ Available online: November 10, 2021}

Key words:

Triacylglycerides, lipid, starvation, stress, biodiesel

\begin{abstract}
This article investigates the induction of lipid synthesis in unicellular green algae Scenedesmus quadricauda UT4, Scenedesmus armatus UT39, Ankistrodesmus falcatus UT20, and Chlorococcum macrostigmatum UT4 under the influence of various stress factors, such as nitrogen starvation, salt, and $\mathrm{pH}$. In this study, the accumulation of neutral lipids of microalgae lasted up to 40 hours under incubation without a nitrogen medium, and they accounted for $39.8 \%-54.2 \%$ of the total dry mass of microalgae. In the presence of 400 and $600 \mathrm{mM}$ $\mathrm{NaCl}$, the highest lipid accumulation of C. macrostigmatum UT4 was $55.5 \%-65.3 \%$, and using pH 4.0 caused the highest accumulation of microalgae lipids $(30.6 \%-63.7 \%)$ that was observed at 18 hours of incubation. From the data obtained, we can conclude that $C$. macrostigmatum UT4 under study is a potential producer of lipids.
\end{abstract}

\section{INTRODUCTION}

The global energy industry is, to a large extent, dependent on fossil energy resources, including oil, coal, and gas. While the demand for these energy carriers is increasing every year, their reserves are steadily decreasing. Therefore, there is growing interest in alternative renewable energy sources that can ensure stable energy production for an indefinite period. Among all known sources, microalgae show high potential for biodiesel production due to their many advantages, such as a higher level of lipid productivity than that of plants and the need for relatively small amounts of agricultural land.

Lipids that carry out essential functions in living organisms can be divided into two key groups: nonpolar or neutral (acylglycerols, wax esters, etc.) and polar (phospholipids and glycolipids) lipids. Polar lipids are important structural components of cell membranes and organoids. Along with their structural function, polar lipids carry out a signal function by signaling either molecules or their predecessors. Among neutral (nonpolar) lipids, triacylglycerols (TAGs) are the most widely distributed group of

*Corresponding Author

Shakirov Zair Saatovich, Institute of Microbiology, Uzbekistan Academy of Sciences, Tashkent, Republic of Uzbekistan.

E-mail:zsshakirov@gmail.com storage substances that are easily involved in catabolism to obtain the necessary energy for the cell [1]. Notably, the conditions that are conducive to the accumulation of TAGs are stress factors for microalgae: they obstruct cell division and slow down culture growth (biomass accumulation), which contradict the task of obtaining the maximum amount of microalgae biomass that is enriched in compounds that are valuable to humans [2,3]. The solution to this nontrivial problem is vital for photobiotechnology that is based on the cultivation of microalgae, making it relevant to study the physiology of the induction of synthesis and the dynamics of the TAGs' content in microalgae that are under various stressors [4]. The accumulation of TAGs by microalgae cells is a two-step process. While all the elements that are necessary for culture growth are present in the medium, the cells of the microalgae divide rapidly, and the biosynthesis of the membrane, including chloroplast lipids, prevails [5]. When one of the growth factors transitions to the limiting one (e.g., when nitrogen is exhausted in the medium) against the background of the continuing carbon dioxide fixation during photosynthesis, the so-called "lipogenic phase," which is characterized by either the slowing down or the stopping of cell division, occurs. This seldom reduces the photosynthetic apparatus and the accumulation of neutral lipids, particularly TAGs and nonpolar carotenoids [2]. In this situation, energy-rich substances, reducing equivalents, and carbohydrates that formed during photosynthesis are primarily 
utilized in the course of biosynthesis of the nitrogen-free storage compounds, mainly TAGs. At the same time, TAG biosynthesis reactions provide an efficient drain for excess photoassimilates. Synthesized de novo TAGs are deposited in the form of either cytoplasmic oleosomes or lipid globules. In some cases, the formation of lipid globules is observed in the intertilakoid space [6]. In optimal growth conditions, microalgae mainly synthesize the lipids of membranes, the content of which reaches $5 \%-20 \%$ of the dry cell weight. On the contrary, under stressful conditions, the lipid metabolism of microalgae shifts toward the synthesis of neutral lipids [7].

\section{MATERIALS AND METHODS}

\subsection{Strains of Microalgae}

The objects of this study were strains of unicellular green algae, including Scenedesmus quadricauda UT4, Scenedesmus armatus UT39, Ankistrodesmus falcatus UT20, and Chlorococcum macrostigmatum UT4 [8,9].

\subsection{Growth Conditions}

Microalgae cultures were grown under sterile optimal conditions in a modified Chu-13 medium for 7 days at $28^{\circ} \mathrm{C}$ with carbon dioxide that was supplied by blown air that contained $2 \% \mathrm{CO}_{2}$ and with continuous illumination by fluorescent white light $(200 \mu \mathrm{mol}$ photons $\left.\mathrm{m}^{-2} \cdot \mathrm{s}^{-1}\right)[10,11]$. Grown microalgae were collected by centrifugation at $3,000 \mathrm{~g}$ for 20 minutes, and the resulting biomass was resuspended in distilled water to remove any residual growth medium. After 10 minutes, the microalgae biomass was again collected by centrifugation (3,000 g for 20 minutes) and used to study the effect of stress factors (nitrogen starvation, salt, and $\mathrm{pH}$ ) on the induction of the lipids' synthesis. The study of the influence of the stress factors of microalgae was carried out with a modified Chu-13 medium without nitrogen, containing $400 \mathrm{mM}$ and 600 $\mathrm{mM} \mathrm{NaCl}$, and a medium with $\mathrm{pH} 4.0$ and 9.0.

\subsection{Lipid Extraction}

Extraction and determination of the intracellular lipids of the microalgae were conducted according to the standard protocols in previous literature $[12,13]$. A dry biomass of algae was added to a glass of sand at a ratio of 1:4, and the mixture was comminuted until smooth. Methanol containing 10\% Dimethyl sulfoxide (volume fraction) was added to the biomass, and the mixture was stirred for 1 hour at room temperature. The mixture was then centrifuged (3,000 $\mathrm{g}$ for 10 minutes), and the supernatant was removed. The residua were reextracted thrice with a mixture of hexane and diethyl ether (1:1, volume ratio) for 30 minutes. Each time, the mixture was shaken and then centrifuged for 10 minutes at 3,000 g, and the upper organic layer was collected. The organic phases were then combined, evaporated until dry, and weighed for microbalance.

\subsection{Statistical Analysis}

The experiments were carried out thrice. The means were expressed as mean \pm SE. The means and standard deviations of the current research study results were calculated using MS Excel Software.

\section{RESULTS AND DISCUSSION}

\subsection{Nitrogen Starvation}

The goal of our research is to study the effects of stress factors, such as nitrogen starvation, salinity, and $\mathrm{pH}$, on lipid accumulation in microalgae cells.

A microalgae biomass was grown under optimal conditions, transferred to a Chu-13 nitrogen-free medium, and incubated for 72 hours. As shown by the results of the experiments, an increase in the accumulation of neutral lipids (TAGs) in the cells of the microalgae A. falcatus UT20, S. quadricauda UT4, and $S$. armatus UT39 lasted for up to 40 hours of incubation. Further incubation ( 72 hours) led to a significant decrease in lipids, regardless of microalgae species (Fig. 1). The highest lipid biosynthesis $(59.7 \%)$ in C. macrostigmatum UT4 cultures was observed after 60 hours of incubation, i.e., with nitrogen starvation, and the lipid accumulation increased nearly three times in size compared to the original culture (22.1\%). Notably, the green color of the microalgae suspension after 60 hours of incubation became yellowish. This meant that, during incubation, the cells of $C$. macrostigmatum UT4 accumulated the largest amount of lipids (Fig. 2). With nitrogen deficiency, the risk of photooxidative death of microalgae cells is significantly increased due to the inhibition of biosynthetic processes in cells, in which photoassimilates are utilized. In such situations, a number of microalgae observe the induction of synthesis of energy-rich TAGs, which accumulate in large quantities. TAGs' biosynthesis reactions seem to provide

an efficient drain for excess photoassimilates and thereby reduce the risk of formation of reactive oxygen species and photooxidative

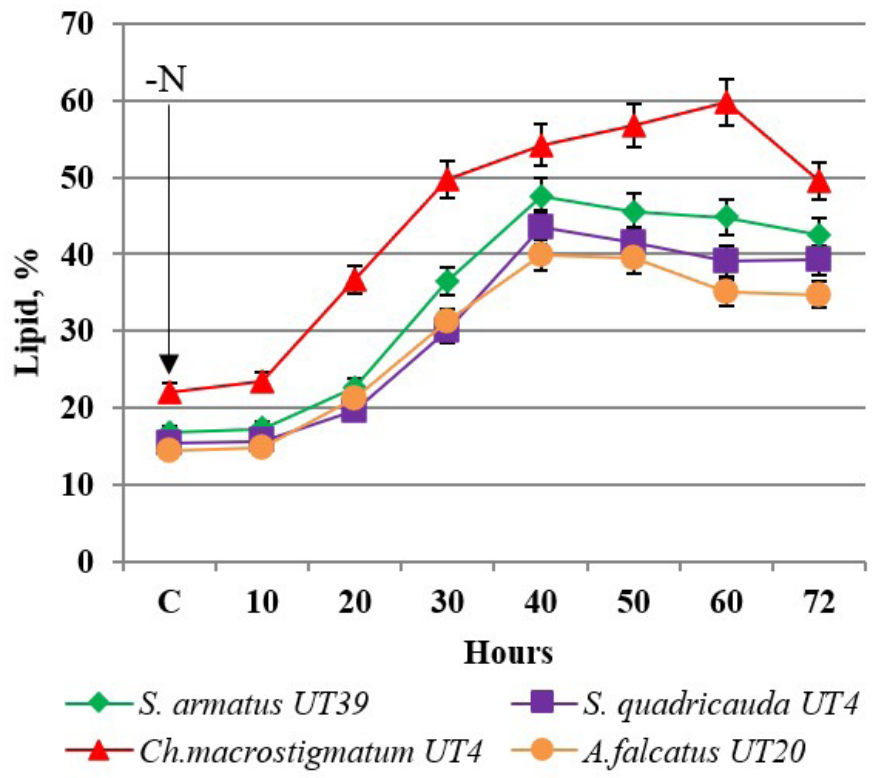

Figure 1. The accumulation of lipids in microalgae with nitrogen starvation. 

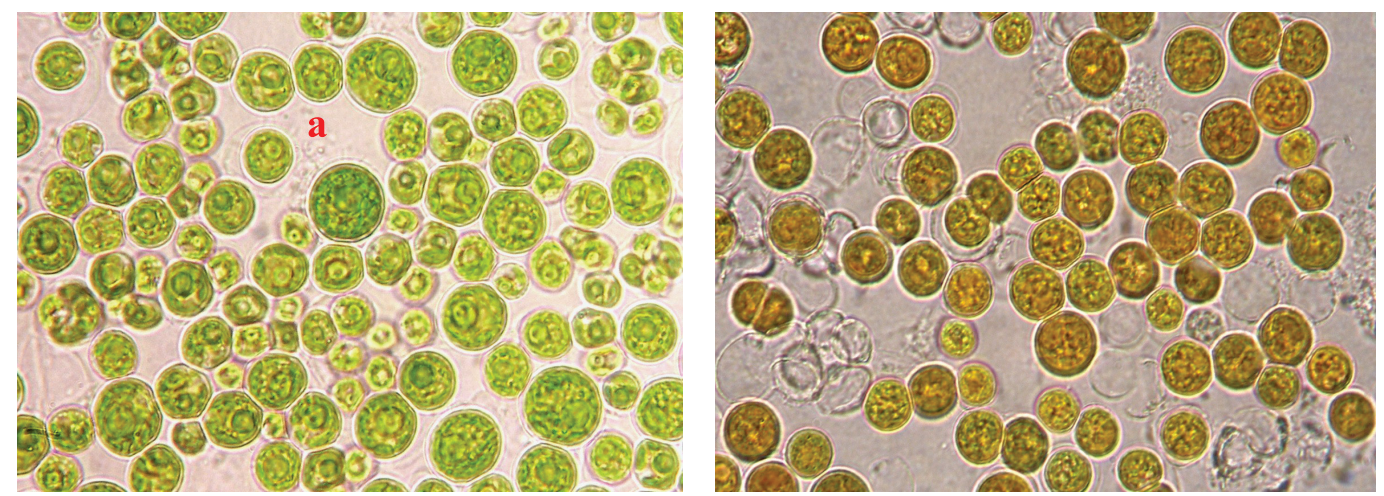

Figure 2. Cells of $C$. macrostigmatum UT4 cultures incubated without nitrogen medium depending on time: (a) cells of the original culture of C. macrostigmatum UT4 and (b) after 60 hours of incubation.

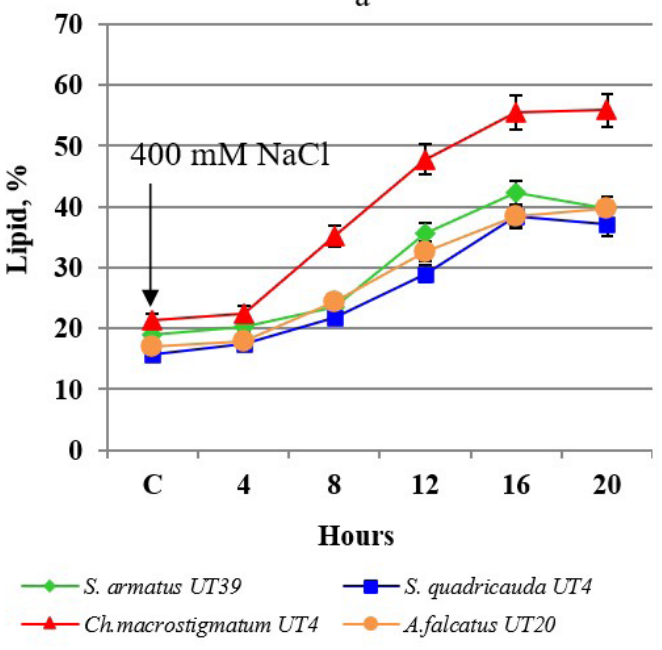

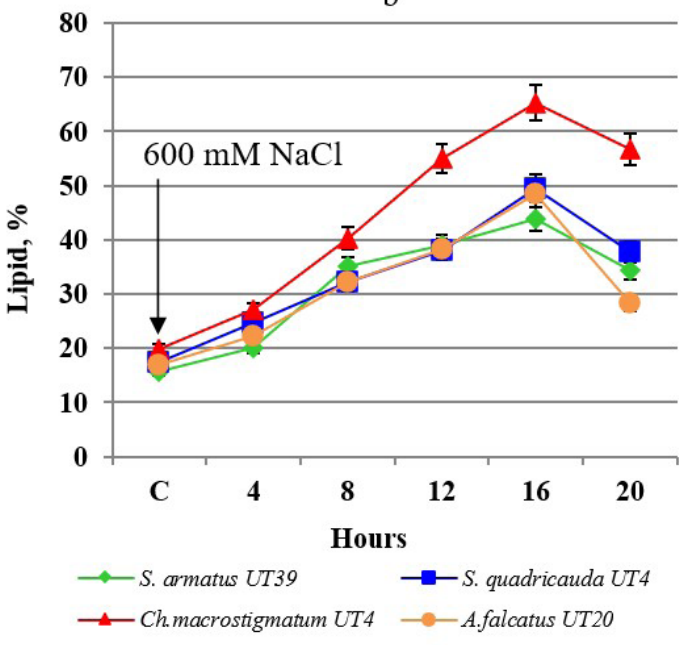

Figure 3. Effect of salinity of (a) $400 \mathrm{mM}$ and (b) $600 \mathrm{mM} \mathrm{NaCl}$ on the lipid content of microalgae.

damage to microalgae cells [6,14]. Thus, with either a lack or complete absence of nitrogen, cell division stops, and TAGs accumulate in cells, which consequently continue to increase in size [15]. As a result, the content of TAGs in microalgae cells can increase two or more times [16].

\subsection{Salt Stress}

The microalgae biomass was introduced into a Chu- 13 medium containing 400 and $600 \mathrm{mM} \mathrm{NaCl}$, and incubation was carried out under optimal conditions for 20 hours. Studies have shown that, in the initial incubation stage of $C$. macrostigmatum UT4, A. falcatus UT20, S. quadricauda UT4, and S. armatus UT39 cultures on a medium containing $400 \mathrm{mM} \mathrm{NaCl}$, lipid accumulation ranged from $17.4 \%$ to $25 \%$ of the total dry biomass (Fig. 3). A further increase in incubation up to 16 hours led to a significantly greater accumulation of microalgae lipids. In particular, in C. macrostigmatum UT4, lipid formation was three times greater than what it was in the initial concentration. After salinization with $600 \mathrm{mM} \mathrm{NaCl}$, the induction of lipid biosynthesis of microalgae began immediately after incubation. The highest accumulation of lipids (43.8\%-65.3\%) was established at 16 hours of incubation, which was the case in the previous experiment. At the same time, an increase in incubation up to 20 hours led to a decrease in the concentration of lipids in the studied cultures $(28.2 \%-56.7 \%)$. Ruangsomboon [17] found that algae can tolerate lower $\mathrm{NaCl}$ concentrations and grow normally. However, with increasing $\mathrm{NaCl}$ concentration, chlorophyll degradation occurs, which leads to cell death. In the work of Takagi et al. [18] on Dunaliella tertiolecta cultures, an increase in the total content of lipids was observed with an increase in the concentration of $\mathrm{NaCl}$ in the medium, from 0.5 to $1.0 \mathrm{M}$. An increase in the content of TAGs along with an increase in the salinity of the cultivation medium to $40 \mathrm{~g} / \mathrm{l}$ was also characteristic of marine microalgae of the genus Nannochloropsis [19].

\section{3. pH Stress}

To study the effect of $\mathrm{pH}$ on lipid synthesis, microalgae were incubated in a Chu- 13 medium at $\mathrm{pH} 4.0$ and 9.0 for 24 hours. As shown by our research, after a 6-hour incubation at $\mathrm{pH} 4.0$, the lipid content of $C$. macrostigmatum UT4 and S. armatus UT39 increased 1.3 times in relation to the initial state. High induction 

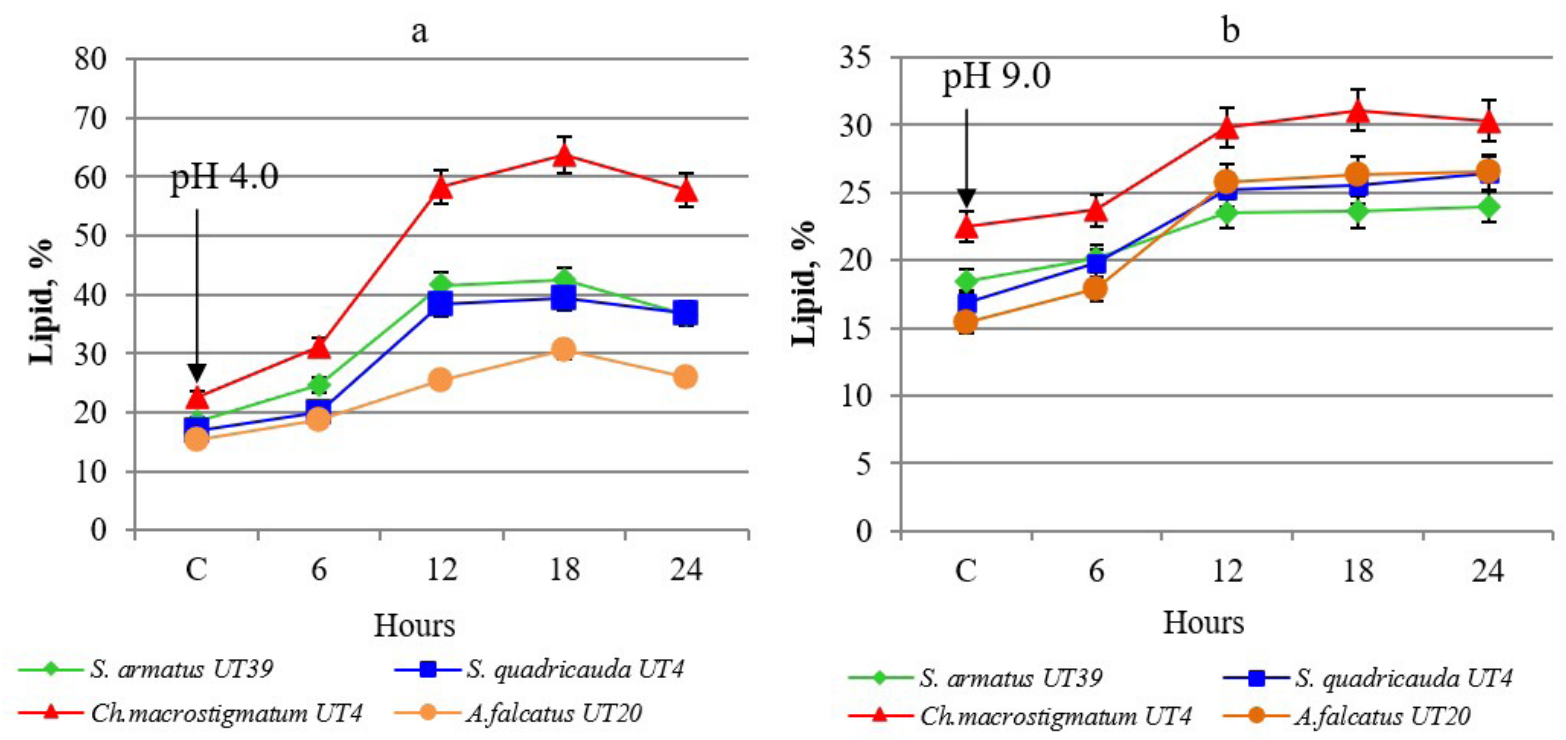

Figure 4. Effect of (a) $\mathrm{pH} 4.0$ and (b) $\mathrm{pH} 9.0$ on the content of lipids in microalgae.

of lipid synthesis of microalgae was observed in the range of 12 18 hours of incubation. The highest lipid accumulation $(63.7 \%)$ was observed in C. macrostigmatum UT4. Further incubation of microalgae for up to 24 hours resulted in a slight loss of lipid content (Fig. 4). During the incubation of microalgae in nutrient media at $\mathrm{pH}$ 9.0, lower lipids were formed compared to those that had formed at $\mathrm{pH}$ 4.0. In particular, in cells of $C$. macrostigmatum UT4 during incubation from 12 to 18 hours, lipid accumulation was two times lower than what was seen at $\mathrm{pH}$ 4.0. Tatsuzawa et al. [20] noted that Chlamydomonas sp., when isolated from an acidic volcanic lake at $\mathrm{pH} 1$, showed an increase in TAG accumulation in cells. Several authors have reported the effect of $\mathrm{pH}$ in different genera of both freshwater and marine microalgae (Thalassiosira pseudonana, Chlorella pyrenoidosa), namely on the growth rate and photosynthetic productivity from $\mathrm{pH}$ values of 5.7, 6.5, and 8.3-8.8; nonetheless, above $\mathrm{pH} 9.0$, growth could not be maintained $[21,22]$.

Thus, under stress conditions in microalgae cells, depending on the time of incubation, lipid accumulation significantly increases. Among the studied cultures, C. macrostigmatum UT4 was more efficient in the synthesis of lipids under stressful conditions.

\section{CONCLUSION}

From the data obtained, we can conclude that $C$. macrostigmatum UT4 under study is a potential producer of oils. Currently, there is increased interest in the synthesis of TAGs of microalgae induction due to being recognized as a promising raw material for the production of third and fourth generations of biodiesel fuel $[6,23,24]$.

The most important advantage of new types of fuel that are produced from TAGs of microalgae is that they are ecologically clean fuels, the burning of which does not lead to an increase in the concentration of $\mathrm{CO}_{2}$ in the atmosphere. Also important is that the production of biofuels from TAGs of microalgae does not threaten food security because the cultivation of microalgae does not occupy the arable land that is necessary for growing crops $[25,26]$.

The accumulation of TAGs in unicellular microalgae is regulated by a number of stressful environmental factors. That is, TAGs are most intensively synthesized against the background of deficiency of nitrogen and during salinization. They are stored by cytoplasmic oleosomes and, under normal conditions, are reused for the synthesis of polar lipids of microalgal membranes.

\section{ACKNOWLEDGMENTS}

This work was financially supported by a project grant (FAF6-T328) from the Committee for Coordination of Science and Technologies Development of the Republic of Uzbekistan, Tashkent. The authors are grateful for the financial support.

\section{ETHICAL APPROVAL}

This study does not involve experiments on animals or human subjects.

\section{CONFLICTS OF INTEREST}

The authors declare no conflicts of interest.

\section{AUTHOR CONTRIBUTIONS}

All authors made substantial contributions to conception and design, acquisition of data, or analysis and interpretation of data; took part in drafting the article or revising it critically for important intellectual content; agreed to submit to the current journal; gave final approval of the version to be published; and agree to be accountable for all aspects of the work. All the authors are eligible to be an author as per the international committee of medical journal editors (ICMJE) requirements/guidelines. 


\section{REFERENCES}

1. Gurr MI, Harwood JL, Frayn KN. Lipid biochemistry: an introduction. 5th edition, Blackwell Science, Oxford, UK, 2002.

2. Leman J. Oleaginous microorganisms: an assessment of the potential. Adv Appl Microbiol 1997;43:195-244.

3. Mayzaud P, Chanut J, Ackman R. Seasonal changes of the biochemical composition of marine particulate matter with special reference to fatty acids and sterols. Mar Ecol Progr Ser 1989;56:189-204.

4. Rai MP, Gautom T, Sharma N. Effect of salinity, pH, light intensity on growth and lipid production of microalgae for bioenergy application. Online J Biol Sci 2015;15(4):260-67.

5. Zhang H, Zhang GY, Wen XB, Geng YH, Li YG. Effects of pH on the photosynthesis, growth and lipid production of Chlorella sp. XQ200419. Acta Hydrobiol Sin 2014;38(6):1084-91.

6. Hu Q, Sommerfeld M, Jarvis E, Ghirardi M, Posewitz M, Seibert M, et al. Microalgal triacylglycerols as feedstocks for biofuel production: perspectives and advances. Plant J 2008;54:621-39.

7. Guschina IA, Harwood JL. Lipids and lipid metabolism in eukaryotic algae. Prog Lipid Res 2006;45:160-86.

8. Shakirov ZS, Safarov IV, Kadirova GK, Khujamshukurov NA. Isolation and identification of lipid-producing microalgae of Uzbekistan. Environ Sci Indian J 2014;9:405-9.

9. Safarov IV, Abdullaev AK, Khujamshukurov NA, Shakirov ZS. Influence of temperature and $\mathrm{CO}_{2}$ on the growth and accumulation oil of microalgae. Br J Appl Sci Technol 2015;10:1-9.

10. Yamaguchi K, Nakano H, Murakami M, Konosu S, Nakayama O, Kanda M, et al. Lipid composition of a green alga, Botryococcus braunii. Agric Biol Chem 1987;51:493-98.

11. Largeau C, Casadevall E, Berkalo VC, Dhamliencourt P. Sites of accumulation and composition of hydrocarbons in Botryococcus braunii. Phytochemistry, 1980;19(6):1043-51.

12. Bligh EG, Dyer WJ. A rapid method for total lipid extraction and purification. Can J Biochem Physiol 1959;37:911-17.

13. Bigognoa C, Khozin-Goldberg I, Boussiba S, Vonshaka A, Cohen Z. Lipid and fatty acid composition of the green oleaginous alga Parietochloris incisa, the richest plant source of arachidonic acid. Phytochemistry 2002;60:497-503.

14. Lemoine Y, Schoefs B. Secondary ketocarotenoid astaxanthin biosynthesis in algae: a multifunctional response to stress. Photosynth Res 2010;106:155-77.

15. Meng X, Yang J, Xu X, Zhang L, Nie Q, Xian M. Biodiesel production from oleaginous microorganisms. Renew Energ 2009;34:1-5.
16. Thompson G. Lipids and membrane function in green algae. Biochim Biophys Acta 1996;1302:17-45.

17. Ruangsomboon S. Effect of light, nutrient, cultivation time and salinity on lipid production of newly isolated strain of the green microalga, Botryococcus braunii KMITL 2. Bioresource Technol 2012;109:26165 .

18. Takagi M, Karseno, Yoshida T. Effect of salt concentration on intracellular accumulation of lipids and triacylglyceride in marine microalgae dunaliella cells. J Biosci Bioeng 2006;101:223-26.

19. Pal D, Khozin-Goldberg I, Cohen Z, Boussiba S. The effect of light, salinity, and nitrogen availability on lipid production by Nannochloropsis sp. Appl Microbiol Biotechnol 2011;90:1429-41.

20. Tatsuzawa H, Takizawa E, Wada M, Yamamoto Y. Fatty acid and lipid composition of the acidophilic green alga Chlamydomonas sp. J Phycol 1996;32:598-601.

21. Chen CY, Durbin EG. Effects of $\mathrm{pH}$ on the growth and carbon uptake of marine phytoplankton. Mar Ecol Prog Ser 1994;109: 89-94.

22. Tan XB, Zhang YL, Yang LB, Chu HQ, Guo J. Outdoor cultures of Chlorella pyrenoidosa in the effluent of anaerobically digested activated sludge: the effects of $\mathrm{pH}$ and free ammonia. Bioresour Technol 2016;200:606-15.

23. Lü J, Sheahan C, Fu P. Metabolic engineering of algae for fourth generation biofuels production. Energy Environ Sci 2011;4:2451-66.

24. Mutanda T, Ramesh D, Karthikeyan S, Kumari S, Anandraj A, Bux F. Bioprospecting for hyper-lipid producing microalgal strains for sustainable biofuel production. Bioresour Technol 2011;102:57-70.

25. Pittman JK, Dean AP, Osundeko O. The potential of sustainable algal biofuel production using wastewater resources. Bioresour Technol 2011;102:17-25.

26. Singh A, Nigam PS, Murphy JD. Mechanism and challenges in commercialisation of algal biofuels. Bioresour Technol 2011;102: 26-34.

How to cite this article:

Shakirov ZS, Khalilov IM, Khujamshukurov NA. Stress factors'effects on the induction of lipid synthesis of microalgae. J Appl Biol Biotech 2021; 9(06):149-153. 